\title{
Dados científicos abertos: identificando o papel das políticas de gestão e das agências de fomento
}

\section{Open scientific data: identifying the role of management policies and development agencies}

\author{
Gabriela Monteiro ${ }^{1}$, Elaine Rosangela de Oliveira Lucas ${ }^{1}$ \\ ${ }^{1}$ Universidade Federal de Santa Catarina, Florianópolis, SC, Brasil
}

Autor para correspondência/Mail to: Gabriela Monteiro, gabriela.monteiro@udesc.br

Financiamento/Funding: Fundação Araucária e Governo do Estado do Paraná

Copyright (c) 2019 Monteiro \& Lucas. Todo o conteúdo da Revista (incluindo-se instruções, política editorial e modelos) está sob uma licença Creative Commons Atribuição-NãoComercial-Compartilhalgual 3.0 Não Adaptada. Ao serem publicados por esta Revista, os artigos são de livre uso em ambientes educacionais, de pesquisa e não comerciais, com atribuição de autoria obrigatória. Mais informações em http://revistas.ufpr.br/atoz/about/submissions\#copyrightNotice.

\begin{abstract}
Resumo
As transformações das práticas científicas apontam que a ciência vive um momento de atenção ao trabalho colaborativo e ao compartilhamento de dados de pesquisa, com o intuito de acelerar a construção de novos conhecimentos e aumentar a eficiência dos investimentos. Agências de fomento internacionais e brasileiras já iniciaram recomendações e exigências para que os projetos financiados incluam um plano de gestão de dados de pesquisa para o compartilhamento em acesso aberto. Em curto ou médio prazo, cada universidade ou centro de pesquisa precisará estar preparado para atender às exigências dos órgãos financiadores. Assim, esta pesquisa tem o objetivo principal de desenvolver um mapeamento das principais políticas institucionais das agências de fomento nacionais e internacionais na promoção do acesso aberto aos dados de pesquisas científicas desenvolvidas no Brasil. A revisão inicial da literatura contempla os temas da comunicação científica e do movimento de acesso aberto e ainda conceitos sobre dados científicos abertos e uma breve contextualização do fomento à pesquisa científica no Brasil. Com relação aos procedimentos metodológicos delineados, a pesquisa é considerada social aplicada no que ser refere a sua finalidade. Quanto ao objetivo central, terá característica exploratória e descritiva e, em relação aos procedimentos técnicos para obtenção dos dados, será realizada pesquisa bibliográfica-documental. A coleta e o tratamento dos dados para identificação das agências de fomento que farão parte da pesquisa documental foi iniciada em abril deste ano. Os dados foram obtidos com base na lista de bolsistas de Produtividade em Pesquisa do Conselho Nacional de Desenvolvimento Científico e Tecnológico vigentes em julho de 2017 e por meio da extração de informações sobre o financiamento de seus projetos, constantes na base de currículos acadêmicos da Plataforma Lattes. A pesquisa encontra-se na fase de submissão ao exame de qualificação.
\end{abstract}

Palavras-chave: Dados Científicos Abertos; Agências de Fomento; Políticas de Dados Abertos; Gestão de Dados de Pesquisa.

\begin{abstract}
The transformations of scientific practices point out that science lives a moment of attention to collaborative work and the sharing of research data, in order to accelerate the construction of new knowledge and increase the efficiency of investments. International and Brazilian development agencies have already initiated recommendations and requirements for funded projects to include a research data management plan for open access sharing. In the short or medium term, each university or research center will need to be prepared to meet the requirements of funding agencies. Thus, this research has the main objective of developing a mapping of the main institutional policies of national and international development agencies in the promotion of open access to the data of scientific research developed in Brazil. The initial review of the literature contemplates the themes of scientific communication and the open access movement, as well as concepts about open scientific data and a brief contextualization of the promotion of scientific research in Brazil. Regarding the methodological procedures outlined, the research is considered social applied in what refers to its purpose. Regarding the central objective, it will have an exploratory and descriptive characteristic and, in relation to the technical procedures to obtain the data, a bibliographical-documentary research will be carried out. The collection and processing of the data to identify the development agencies that will be part of the documentary research began in April of this year. The data were obtained based on the list of Research Productivity Scholarships of the National Council of Scientific and Technological Development in July 2017 and through the extraction of information on the financing of their projects, which are based on the academic curriculum of the Plataforma Lattes. The research is in the phase of submission to the qualification examination.
\end{abstract}

Keywords: Open Scientific Data; Agencies of Promotion; Open Data Policy; Research Data Management.

\section{INTRODUÇÃO}

Os conceitos que permeiam o movimento de acesso aberto às publicações científicas se ampliaram para o reconhecimento de que o conhecimento científico é um patrimônio da humanidade. A evolução das práticas científicas aponta que a ciência vive atualmente o chamado "quarto paradigma" ou "e-Science", preocupando-se com o compartilhamento dos dados de pesquisa.

Estudos recentes apontam que agências de fomento brasileiras e internacionais já iniciaram recomendações, ou exigências, para que os projetos financiados incluam um plano de gestão de dados de pesquisa para o compartilhamento em acesso aberto. Considerando que as instituições de pesquisa precisam estar preparadas para atender essas novas regras, surge o problema a ser investigado nesta pesquisa: quais são as principais políticas institucionais das agências de fomento nacionais e internacionais na promoção do acesso aberto aos dados de pesquisas científicas, desenvolvidas no Brasil?

Com a finalidade de possibilitar o enfrentamento do problema de pesquisa foi delineado o seguinte objetivo geral: Desenvolver um mapeamento das principais políticas institucionais das agências de fomento nacionais e 
internacionais na promoção do acesso aberto aos dados de pesquisas científicas desenvolvidas no Brasil. Para atingi-lo serão desenvolvidos os seguintes objetivos específicos: a) Identificar as principais agências de fomento, nacionais e internacionais, que financiam pesquisas desenvolvidas no Brasil; b) Analisar as políticas institucionais das agências identificadas para a promoção do acesso aberto aos dados de pesquisa; c) Categorizar as políticas analisadas quanto ao seu caráter mandatório ou recomendatório para o acesso aberto aos dados de pesquisa; d) Apresentar um infográfico das principais políticas institucionais identificadas, analisadas e categorizadas conforme itens a, b e c.

Esta investigação se justifica em função de alguns aspectos. Inicialmente é preciso considerar que o desenvolvimento científico de um país é fundamental para a construção de uma sociedade alicerçada no conhecimento, auxiliando no crescimento econômico e na diminuição das desigualdades e exclusões sociais.

As práticas da ciência contemporânea revelam um momento de preocupação com a abertura e a transparência não apenas dos seus resultados, mas também dos dados brutos das pesquisas, possibilitando o compartilhamento e a colaboração no trabalho científico e aumentando a eficiência e a efetividade dos recursos econômicos e sociais investidos.

O movimento de acesso aberto às publicações científicas iniciado no final da década de 1980 e início dos anos de 1990 demonstra expansão, ampliando as diretrizes declaradas por instituições internacionais e nacionais para a abertura dos dados de pesquisa, reconhecendo o potencial informacional contido na ideia de ciência aberta e colaborativa.

Neste cenário de transição, pesquisadores, instituições acadêmicas e agências de fomento também começam a compreender a necessidade de tratar, preservar, gerenciar e compartilhar os dados de pesquisa.

O Comitê de Política Científica e Tecnológica da Organization for Economic Cooperation and Development (OECD) reuniu-se em 2004 e emitiu uma Declaração sobre acesso a dados de pesquisa com financiamento público, em que se comprometeram governos de 35 países da Europa, América do Norte, África, Ásia e Oceania. A Declaração reconhece que a divulgação dos dados de pesquisa pode aumentar a participação dos países em desenvolvimento no sistema científico global, contribuindo para o desenvolvimento social e econômico e facilitando a formação de novos pesquisadores. Apresenta ainda uma lista de princípios que fundamentam o estabelecimento do acesso aberto aos dados de pesquisa.

No Brasil o tema ainda é recente e poucas são as iniciativas para a promoção da publicação de dados de pesquisa. A Fundação de Amparo à Pesquisa do Estado de São Paulo (FAPESP) passou a exigir em 2017 a apresentação de um Plano de Gestão de Dados para os projetos submetidos aos seus programas de fomento. A Fundação reconhece a importância da gestão adequada dos dados de pesquisa, considerando necessário que os dados resultantes de projetos financiados por ela sejam gerenciados e compartilhados.

Em curto ou médio prazo cada universidade ou centro de pesquisa precisará estar preparado para atender às exigências dos órgãos financiadores, criando planos de gestão de dados de pesquisa, para apresentação já na submissão dos projetos que pretendem financiamento.

Assim, diante desse contexto, este projeto visa à realização de uma pesquisa que terá como tema central o acesso aberto aos dados de pesquisas científicas desenvolvidas no Brasil, com foco nas políticas institucionais das agências de fomento nacionais e internacionais na promoção desse acesso.

\section{REVISÃO DE LITERATURA}

Após uma exploração inicial acerca do problema de pesquisa, foram definidos os seguintes assuntos que merecem atenção no processo de revisão da literatura: comunicação científica e movimento de acesso aberto, dados científicos abertos e fomento à pesquisa científica no Brasil.

\section{Comunicação científica e movimento de acesso aberto}

A comunicação científica se caracteriza pelo intercâmbio contínuo de informações entre os membros de uma comunidade científica, integrando a estrutura social que esta comunidade se constitui. A comunicação científica engloba as atividades de produção, disseminação e uso da informação desde o momento da concepção de uma ideia por um cientista até a aceitação e a visibilidade dos resultados (Targino, 2000).

De acordo com Baptista, Costa, Kuramoto, \& Rodrigues (2007), dentre os canais utilizados pela comunidade científica, o periódico científico tem sido considerado o veículo formal mais importante para consolidação e disseminação dos resultados das pesquisas realizadas por estudiosos de todo o mundo. Mueller (2006) afirma que há uma hierarquia entre os diferentes veículos que podem ser usados para comunicar o conhecimento científico e que, embora varie conforme a área, o periódico indexado costuma ser o veículo mais prestigiado. Da mesma forma, há graus diferentes de prestígios às editoras, à língua que usam e às bases de dados que os indexam. 
Leite (2009) afirma que a criação dos periódicos científicos eletrônicos na década de 1990 foi considerada inovadora e um dos grandes avanços tecnológicos na comunicação científica, entretanto pouco alterou a hegemonia dos editores científicos, que conduzem o cerne do sistema de publicações científicas. Leite (2009, p. 14) ainda salienta que apenas recentemente este sistema tradicional de publicação científica "em que editores comerciais retêm os direitos autorais, atribuem preços excessivos e impõem barreiras ao acesso às publicações dos resultados de pesquisas financiadas com recursos públicos", passou a ser questionado.

Sendo a informação científica fundamental para o desenvolvimento científico e tecnológico de um país, Kuramoto (2006, p. 92) afirma que:

É importante ressaltar que as pesquisas científicas, em sua maioria, são financiadas pelo Estado, portanto, com recursos públicos. Do ponto de vista ético, os resultados dessas pesquisas deveriam ser de livre acesso. Não é isso, entretanto, o que acontece no sistema de comunicação científica tradicional. O pesquisador ou qualquer outro cidadão, para ter acesso àquilo que foi produzido com o apoio do Estado, precisará pagar pela assinatura de uma publicação científica. Trata-se de uma situação paradoxal, pois o Estado, para promover o acesso àquilo que produz, é obrigado a arcar com os custos de manutenção das coleções das revistas em que são publicados os resultados de sua produção científica [...].

Nessa direção, Mueller (2006) aponta a chamada "crise dos periódicos", ocorrida nos anos 80 e 90 e disparada pela impossibilidade das bibliotecas universitárias e de pesquisa estadunidense manterem suas coleções de periódicos atualizadas em função dos altos custos impostos pelas editoras comerciais, como o gatilho para o surgimento de iniciativas concretas de promoção ao acesso livre aos resultados acadêmicos.

Alguns dos acontecimentos históricos recebem destaque na literatura sobre o tema, uma vez que diferentes instituições de pesquisa aderiram ao movimento por meio do estabelecimento de declarações. A Budapest Open Access Initiative (BOAI), documento originado da reunião promovida pela Open Society Institute (OSI), realizada em Budapeste em 2001, constitui uma declaração de princípios fundamentais para o movimento de acesso aberto. A Declaração de Budapest define que:

Acesso aberto à literatura científica revisada por pares significa a disponibilidade livre na Internet, permitindo a qualquer usuário ler, fazer download, copiar, distribuir, imprimir, pesquisar ou referenciar o texto integral desses artigos, recolhe-los para indexação, introduzi-los como dados em software, ou usá-los para outro qualquer fim legal, sem barreiras financeiras, legais ou técnicas que não sejam inseparáveis ao próprio acesso a uma conexão à Internet. As únicas restrições de reprodução ou distribuição e o único papel para o direito autoral neste domínio é dar aos autores o controle sobre a integridade do seu trabalho e o direito de ser devidamente reconhecido e citado (Budapest Open Access Initiative, 2002).

A Declaração de Budapeste ainda definiu duas estratégias para que a disponibilização efetiva da literatura científica ocorra: a via verde (green road) e a via dourada (golden road). De acordo com Alves (2008), a primeira estratégia é a do auto arquivamento (via verde), que trata do arquivamento realizado pelos próprios autores dos artigos científicos já publicados ou aceitos para publicação, obtendo autorização dos editores que os aceitaram para disponibilizar em um servidor de arquivo aberto. A segunda estratégia é a via dourada, que abrange periódicos científicos eletrônicos cujo acesso aberto aos seus conteúdos é garantido pelos próprios editores. Outro acontecimento que recebe destaque na história do movimento de acesso aberto é a Declaração de Berlim sobre Acesso Livre ao Conhecimento Ciências e Humanidades, de 2003, que afirmou que o estabelecimento do acesso livre como um procedimento vantajoso requer o empenho ativo de todo e qualquer indivíduo que produza conhecimento científico (Berlin Declaration on Open Access to Knowledge in the Sciences and Humanities, 2003).

No Brasil, o destaque se dá ao Manifesto Brasileiro de Apoio ao Acesso Livre à Informação Científica, lançado pelo Instituto Brasileiro de Informação em Ciência e Tecnologia (IBICT) (2005). O Manifesto apresenta recomendações aos quatro principais grupos de interesse para a informação científica: a comunidade científica, as instituições acadêmicas, as agências de fomento e as editoras comerciais: a) Às instituições acadêmicas afirma que são imperativos a criação e o fomento de repositórios institucionais e temáticos, observando o paradigma do acesso livre; b) Aos pesquisadores recomenda que seja primordial a contribuição para o incremento de conteúdos em repositórios institucionais ou temáticos, além de destacar a obrigatoriedade de depositar em um repositório de acesso livre as publicações que envolvam resultados de pesquisas financiadas com recursos públicos; c) Às agências de fomento, o Manifesto afirma que é necessário o reconhecimento das publicações científicas em repositórios de acesso livre para efeito de avaliação da produção científica; d) Às editoras comerciais, afirma que é imprescindível a concordância com o depósito em repositórios de acesso livre das pesquisas financiadas com recursos públicos; e) Às editoras não comerciais, recomenda que tenham disponível uma versão eletrônica em conformidade com o paradigma do acesso livre e com os padrões da Open Archives Initiative (OAI) (Instituto Brasileiro de Informação em Ciência e Tecnologia, 2005).

Nos últimos anos, foi possível identificar que algumas declarações começaram a incluir a preocupação com o acesso aberto aos dados de pesquisa. De acordo com Sayão e Sales (2014, p. 77), o conceito de acesso aberto, 
considerado crítico para a prática de uma ciência mais aberta, "não está circunscrito às publicações acadêmicas, como os artigos de periódicos; suas demandas avançam para outros conteúdos que incluem, de forma privilegiada, a disponibilização aberta e de forma inteligível de dados de pesquisa".

\section{Dados científicos abertos}

A ideia de ciência aberta tem como um de seus pressupostos a disponibilidade do conhecimento científico para que as pessoas, sendo ou não cientistas, "possam usá-lo, reusá-lo e distribuí-lo sem constrangimentos tecnológicos, econômicos, sociais ou legais" (Sayão \& Sales, 2014, p. 77).

A evolução das práticas científicas aponta que a ciência vive atualmente o chamado "quarto paradigma" ou "e-Science". O primeiro paradigma diz respeito à ciência construída com base na descrição dos fenômenos naturais. O segundo paradigma se constitui numa ciência de descobertas teóricas, modelos e generalizações. Nas últimas décadas tem-se uma ciência baseada nas tecnologias computacionais, permitindo a simulação de fenômenos complexos. E, atualmente, a ciência está se preocupando com o compartilhamento dos dados de pesquisa (Oliveira \& Silva, 2016).

Assim, o quarto paradigma científico se caracteriza pela ciência aberta que tem em sua agenda a ampla disponibilidade e acessibilidade dos dados de pesquisa, com o objetivo de ampliar a utilidade e aplicação desses dados, tornando- se uma ciência transparente que possibilita a reprodutibilidade. Além disso, os pressupostos da ciência aberta visam melhorar a relação custo-eficácia da aplicação dos recursos econômicos e sociais (Curty, 2016).

A Organization for Economic Cooperation and Development (OECD) publicou em 2007 um relatório intitulado OECD Principles and guidelines for access to reserach data from public funding, apresentando princípios e diretrizes como recomendações para políticas governamentais, para instituições de pesquisa e agências de fomento dos países membros sobre o acesso a dados de pesquisa com financiamento público. Entre suas finalidades está "melhorar a eficiência e eficácia do sistema científico global" (Organisation for Economic Co-Operation and Development, 2007, p. 13).

Em um estudo publicado em 2013, Sayão e Sales analisaram além do Relatório da OECD citado acima, outros dois relatórios considerados por eles primordiais para a gestão e compartilhamento de dados de pesquisa em âmbito nacional e internacional: Long-lived digital data collections: enabling research and education in the 21st Century, publicado pela agência norte-americana National Science Foundation (NSF) em 2005; e A comparative study of the international approaches to enabling the sharing of research data, publicado em 2008 pela Digital Data Curation $(D C C)$. O objetivo dos autores com a análise desses relatórios foi contribuir para a construção de um modelo de curadoria digital de dados de pesquisa para o Brasil, alinhando as instâncias política, organizacional, coleções de dados, pesquisa, infraestrutura e tecnologia, padronização, formação de recursos humanos, sustentabilidade econômica, instâncias social, legal e ética e serviços.

Essa complexidade que envolve a questão da promoção do acesso aberto aos dados de pesquisa exige ainda algumas conceituações fundamentais. O relatório da OECD (2007), em tradução livre, define dados de pesquisa como:

Registros factuais (escores numéricos, registros textuais, imagens e sons) utilizados como fontes primárias para pesquisa científica e que são comumente aceitos na comunidade científica como necessário para validar resultados da pesquisa. Um conjunto de dados de pesquisa constitui uma representação sistemática e parcial do assunto que está sendo investigado.

Uma característica importante dos dados de pesquisa é a sua natureza heterogênea, uma vez que as mais diversas áreas do conhecimento coletam e produzem dados nas suas investigações. Sayão e Sales (2013, p. 6) apresentam a categorização do relatório da NSF que distingue os dados pela sua natureza ou origem em observacionais, computacionais e experimentais:

Os dados observacionais são dados obtidos de observações diretas, que podem ser associadas a lugares e tempo específicos, como por exemplo, a erupção de determinado vulcão numa data específica, a fotografia de uma supernova, o levantamento as atitudes de uma comunidade. [...] Os dados computacionais, por sua vez, são resultados da execução de modelos computacionais ou de simulações, seja, por exemplo, no domínio da Física Nuclear u para a criação de ambientes virtuais culturais ou educacionais. [...] Os dados experimentais são provenientes de situações controladas em bancadas de laboratórios, como por exemplo, medidas de uma reação química.

Outro aspecto importante a considerar é o tempo de vida dos dados de uma pesquisa. Sayão e Sales (2015) publicaram um Guia de Gestão de Dados de Pesquisa, editado para bibliotecários e pesquisadores, que além de definir conceitos serve de manual para a construção de um Plano de Gestão de Dados, reconhecendo o potencial informacional dos dados de pesquisa para a ciência. Neste guia, os autores apresentam um modelo de referência sobre o ciclo de vida dos dados constituído em oito etapas que se retroalimentam: planejar, coletar, assegurar a qualidade, descrever, preservar, descobrir, integrar e analisar. 
O ponto crucial para essa preocupação com o ciclo de vida dos dados de pesquisa é o reuso dos dados. O conceito de reuso pode ser compreendido "de maneira ampla como uso de dados - normalmente sem explícita permissão para estudos previstos ou não pelo autor original dos dados, por outros pesquisadores" (Sayão \& Sales, 2014, p. 82). Neste sentido encontra-se a importância de planejar a coleta, a descrição e a preservação dos dados de maneira a assegurar a sua qualidade para que outros pesquisadores possam reusa-los, descobrindo novos dados, integrando e analisando os dados já existentes.

\section{Fomento à pesquisa científica no Brasil}

As agências de fomento constituem importantes agentes no desenvolvimento científico do país. Os primeiros movimentos e debates para criação de uma entidade governamental para fomentar este desenvolvimento surgiram na década de 1930, com a sugestão dos integrantes da Academia Brasileira de Letras para a criação de um Conselho de Pesquisas (Conselho Nacional de Desenvolvimento Científico e Tecnológico [CNPq], 2017).

Com o advento da Segunda Guerra Mundial, os países aceleraram suas pesquisas e a montaram estruturas de fomento ao desenvolvimento científico e tecnológico. Em 1951, após um grupo de cientistas da Sociedade Brasileira para o Progresso da Ciência reforçar a necessidade de um aparato institucional para o desenvolvimento da ciência no país e a discussão do assunto no âmbito de várias comissões governamentais, o presidente Eurico Gaspar Dutra sancionou a Lei que criou o Conselho Nacional de Pesquisas (CNPq). As suas finalidades eram promover e estimular o desenvolvimento da investigação científica e tecnológica, mediante a concessão de recursos para pesquisa, formação de pesquisadores e técnicos, cooperação com as universidades brasileiras e intercâmbio com instituições estrangeiras (CNPq, 2017).

No mesmo ano da criação do CNPq, mais precisamente no dia 11 de julho de 1951, foi criada a Campanha (atual Coordenação) Nacional de Aperfeiçoamento de Pessoal de Nível Superior (Capes) para garantir recursos específicos de formação de cientistas e pesquisadores no ambiente acadêmico. Era o início do segundo governo de Getúlio Vargas e a industrialização e a complexidade da administração pública evidenciaram a necessidade de formação de especialistas e pesquisadores nos diferentes ramos de atividade (Coordenação de Aperfeiçoamento de Pessoal de Nível Superior [Capes], 2017; CNPq, 2017).

Na década de 80 dois eventos marcaram a história da institucionalização do fomento à pesquisa no país. Em 1985 foi criado o Ministério da Ciência, Tecnologia e Inovação (MCTI), que recebeu como competências principais a política nacional de pesquisa científica, tecnológica e inovação e o planejamento, coordenação, supervisão e controle das atividades da ciência e tecnologia. Já em 1988, com a promulgação da Constituição da República Federativa do Brasil, foi instituída a função do Estado na promoção e incentivo do desenvolvimento científico, da pesquisa e da capacitação tecnológica (Constituição da República Federativa do Brasil, 1988/2013; CNPq, 2017).

Atualmente, a Estratégia Nacional de Ciência, Tecnologia e Inovação para os anos de 2016 a 2020 (Encti 2016-2020) apresenta a orientação estratégica de médio prazo para a implementação de políticas públicas na área de Ciência, Tecnologia e Inovação (CT\&I). No documento, são destacados o funcionamento do Sistema Nacional de Ciência, Tecnologia e Inovação (SNCTI), os atores que o compõem e as principais tendências e desafios para a promoção, ampliação e modernização da CT\&I no país. O SNCTI é composto por entidades organizadas em três níveis de atuação (Ministério da Ciência, Tecnologia, Inovações e Comunicações [MCTIC], 2016).

No primeiro nível estão os atores políticos. No âmbito do Poder Executivo atua o MCTIC, Coordenando o SNCTI, com a competência legal para a formulação das Políticas Nacionais. No segundo nível estão as agências de fomento à pesquisa científica, apresentando distinções no que se refere a vinculações ministeriais, a grupos de beneficiários de suas ações e à disponibilidade de instrumentos. Além das agências de fomento federais, as Fundações Estaduais de Amparo à Pesquisa (FAPs) também atuam para promover a pesquisa em CT\&I em âmbito regional. Existem atualmente 26 FAPs, distribuídas em 25 estados do Brasil mais o Distrito Federal. No terceiro nível estão os operadores de CT\&I, instituições onde são geradas as inovações, desenvolvidas as tecnologias e realizadas as pesquisas que foram objeto de diretrizes no nível político e de alocações de recursos no nível das Agências de Fomento (MCTIC, 2016; Conselho Nacional das Fundações Estaduais de Amparo à Pesquisa [CONFAP], 2017).

\section{PROCEDIMENTOS METODOLÓGICOS}

A pesquisa social utiliza a metodologia científica para a obtenção de novos conhecimentos no campo da realidade social. A pesquisa aplicada, por sua vez, tem como característica fundamental o interesse na aplicação, utilização e consequências práticas dos conhecimentos. Portanto, quanto à sua finalidade, esta pesquisa é classificada como social aplicada (Gil, 2008).

Quanto ao objetivo central, esta pesquisa caracteriza-se como exploratória e descritiva. Exploratória, pois irá proporcionar a obtenção de novos conhecimentos sobre o assunto investigado, permitindo maior compreensão do tema e contribuindo para encontrar respostas ao problema de pesquisa. E descritiva, pois os dados coletados serão 
registrados, ordenados e interpretados, com a finalidade de atingir os objetivos inicialmente fixados (Prodanov \& Freitas, 2013).

Em relação aos procedimentos técnicos para obtenção dos dados necessários à realização desta pesquisa, temos a pesquisa bibliográfica e a pesquisa documental.

Para o levantamento bibliográfico foram definidas oito bases de dados, a saber: Base de Teses e Dissertações da Coordenação de Aperfeiçoamento de Pessoal de Nível Superior (BDTD/Capes); Networked Digital Library of Theses and Dissertations (NDLTD); Base dos Encontros Nacionais de Pesquisa e Pós-Graduação em Ciência da Informação (BENANCIB); Base de Dados Referenciais de Artigos de Periódicos em Ciência da Informação da Universidade do Paraná (BRAPCI); Library, Information Science E Technology Abstracts (LISTA/EBSCO); Library and Information Science Abstracts (LISA/ProQuest); Web of Science (Clarivate Analytics) e Scopus (Elsevier). Para a busca nestas bases ainda será necessário estabelecer os termos que melhor representam o tema investigado.

A partir das publicações encontradas será realizada a leitura flutuante (Bardin, 2011), com vistas a conhecer os documentos coletados e a realizar uma seleção daqueles que serão pertinentes ao corpus da pesquisa. Após, serão lidas em profundidade as publicações selecionadas, categorizando os principais temas e conceitos teóricos com a finalidade de fundamentar a redação do referencial teórico definitivo que irá orientar os demais passos da pesquisa e a execução dos seus objetivos.

O primeiro objetivo específico desta pesquisa consiste em identificar as principais agências de fomento, nacionais e internacionais, que financiam pesquisas desenvolvidas no Brasil. Antes de detalhar os passos para atingir este primeiro objetivo, é necessário explicar a delimitação feita para possibilitar a identificação das agências de fomento a serem efetivamente pesquisadas. Os dados que serão utilizados para atingi-lo foram gentilmente cedidos pelo Professor Doutor Fábio Castro Gouveia (Fiocruz), que atua na área de Ciência da Informação com ênfase em Estudos Métricos da Informação. Esses dados foram obtidos com base na lista de Bolsistas de Produtividade em Pesquisa do CNPq, vigentes em julho de 2017 e por meio da extração de informações da base de currículos acadêmicos da Plataforma Lattes, utilizando-se a ferramenta scriptLattes. O relatório resultante contém dados sobre os financiadores de 151.481 projetos de pesquisa, registrados por 14.5898 pesquisadores Bolsistas de Produtividade em Pesquisa.

Os dados extraídos da base de currículos acadêmicos da Plataforma Lattes são de preenchimento livre pelos pesquisadores e o formato das informações não está padronizado, contendo as mais variadas formas de identificação das instituições que contribuíram para o desenvolvimento dos projetos de pesquisa e exigindo tratamento e normalização. Assim, para a consecução do objetivo específico inicial será necessária uma padronização das nomenclaturas, com uma consequente classificação e compilação das informações sobre os financiadores, identificando assim as agências com maior representatividade em número de projetos financiados que serão objeto de estudo para o próximo passo da investigação.

O segundo objetivo específico desta pesquisa tem o intuito de analisar as políticas institucionais das agências identificadas para a promoção do acesso aberto aos dados de pesquisa. Para isso serão realizadas tuas etapas: a) Consulta aos Portais Eletrônicos das agências que se destacaram anteriormente, com o objetivo de levantar documentos que caracterizem institucionalmente cada uma das agências quanto às suas finalidades, áreas de atuação e modalidades de fomento; b) Análise de documentos que caracterizem a existência de políticas institucionais formalizadas ou programas específicos para a promoção do acesso aberto aos dados de pesquisa.

O terceiro objetivo específico tem a intenção de categorizar as políticas institucionais quanto ao seu caráter recomendatório ou mandatório para o acesso aberto aos dados de pesquisa. Nesta etapa será realizada a metodologia de Análise de Conteúdo, definida por Bardin (2011, p. 48) como:

Um conjunto de técnicas de análise das comunicações visando obter por procedimentos sistemáticos e objetivos de descrição de conteúdo das mensagens indicadores (quantitativos ou não) que permitam a inferência de conhecimentos relativos às condições de produção/recepção (variáveis inferidas) dessas mensagens.

Esta metodologia é constituída de três etapas: pré-análise, exploração do material e tratamento dos resultados (inferência e interpretação); o que permitirá categorizar e analisar as políticas institucionais contidas nos documentos coletados.

O quarto objetivo específico consiste na construção de um mapa como representação gráfica dos dados coletados e analisados, na forma de um infográfico, contendo as principais políticas institucionais das agências de fomento nacionais e internacionais na promoção do acesso aberto aos dados de pesquisas científicas desenvolvidas no Brasil, concretizando assim, o objetivo geral desta pesquisa.

Por fim, para alinhamento ao objeto da pesquisa, pretende-se disponibilizar os dados e os resultados obtidos no repositório online Figshare9, ferramenta que permite o armazenamento de publicações de pesquisas científicas, comportando diferentes formatos de arquivos e sem restrições ou barreiras de acesso. 


\section{CONSIDERAÇÕES PARCIAIS}

A coleta e o tratamento dos dados para identificação das agências de fomento que farão parte da pesquisa documental foi iniciada em abril deste ano. Para a compilação dos dados que identificam o número de projetos que cada agência de fomento financiou, está sendo utilizado o recurso de Tabela Dinâmica do Programa Excel. A partir do resultado da tabela dinâmica é possível identificar as agências mais representativas em número de financiamentos.

Além dos dados com relação às agências, também está em andamento a busca sistemática nas bases de dados para a conclusão da revisão de literatura. Com os resultados das coletas será possível identificar ainda a evolução da produção da literatura científica sobre dados científicos abertos. No entanto, os dados ainda não estão compilados para apresentação neste momento.

Atualmente a pesquisa encontra-se na fase de submissão ao exame de qualificação.

\section{AGRADECIMENTOS}

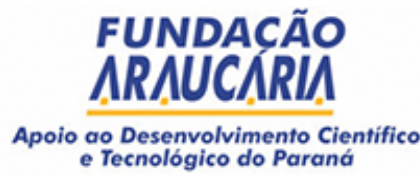

FUNDACÃO

Apoio ao Desenvolvimento Cientifico

e Tecnológico do Parana

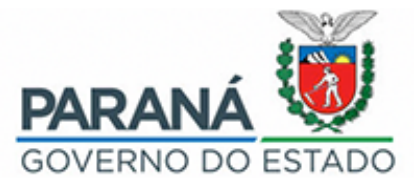

À Fundação Araucária e ao Governo do Estado pelo apoio financeiro para a realização do evento.

\section{REFERÊNCIAS}

Alves, V. B. A. (2008). Open Archives: via verde ou via dourada? Ponto de Acesso, 2(2), 127-137. Recuperado de https://portalseer.ufba.br/index.php/revistaici/article/view/1780

Baptista, A. A., Costa, S. M. de S., Kuramoto, H., \& Rodrigues, E. (2007). Comunicação científica: o papel da Open Archives Initiative no contexto do Acesso Livre. Pesquisa Brasileira Em Ciência da Informação e Biblioteconomia, 2(2), 1-17.

Bardin, L. (2011). Análise de Conteúdo. São Paulo: Edições 70.

Berlin Declaration on Open Access to Knowledge in the Sciences and Humanities. (2003). Berlin. Recuperado de https://openaccess.mpg.de/Berliner-Erklaerung

Budapest Open Access Initiative (BOAI). (2002). Declaração de Budapest. Recuperado de https://www.budapesto penaccessinitiative.org/boai-10-translations/portuguese-brazilian-translation

Conselho Nacional das Fundações Estaduais de Amparo à Pesquisa (CONFAP). (2017). (Brasil). Informações sobre FAPs. Recuperado de http://confap.org.br/news/informacoes-sobre-faps/

Coordenação de Aperfeiçoamento de Pessoal de Nivel Superior (Capes). (2017). Ministério da Educação. Acesso à Informação: Institucional. Recuperado de http://www.capes.gov.br/acessoainformacao/institucional

Conselho Nacional de Desenvolvimento Cientifico e Tecnológico (CNPq). (2017). Ministério da Ciência, Tecnologia, Inovações e Comunicações. Centro de Memória. Recuperado de http://centrodememoria.cnpq.br/cmemoriaindex.html

Constituição da República Federativa do Brasil. (1988). Brasília: Centro de Documentação e Informação (CEDI), 2013. Recuperado de http://www.planalto.gov.br/ccivil_03/constituicao/constituicao.htm

Curty, R. G. A. (2016). As diferentes dimensões do reuso de dados científicos. Tendências da Pesquisa Brasileira em Ciência da Informação, 9(2).

Recuperado de http://www.brapci.inf.br/index.php/article/view/0000022969/9c7f5cf3f928e4a2f342af\%201eecf665e4

Gil, A. C. (2008). Métodos e Técnicas de Pesquisa Social (6a ed.). São Paulo: Atlas.

Instituto Brasileiro de Informação em Ciência e Tecnologia. (2005). Manifesto brasileiro de apoio ao acesso livre à informação científica. Brasília. Recuperado de http://livroaberto.ibict.br/Manifesto.pdf

Kuramoto, H. (2006). Informação científica: proposta de um modelo para o Brasil. Ciência da Informação. Brasília, 35(2), 91-102. Recuperado de http://www.scielo.br/pdf/ci/v35n2/a10v35n2.pdf

Leite, F. C. L. (2009). Como gerenciar e ampliar a visibilidade da informação científica brasileira: Repositórios Institucionais de Acesso Aberto. Brasília: Ibict. 
Ministério da Ciência, Tecnologia, Inovações e Comunicações (MCTIC). (2016) Estratégia Nacional de Ciência, Tecnologia e Inovação 2016-2010. Recuperado de: https://portal.insa.gov.br/images/documentos-oficiais/ENCTIMCTIC-2016- 2022.pdf

Ministério da Ciência, Tecnologia, Inovações e Comunicações (MCTIC). (2017) Institucional. Recuperado de http://www.mctic.gov.br/mctic/opencms/institucional/paginaInstitucional.html

Mueller, S. P. M. (2006). A comunicação científica e o movimento de acesso livre ao conhecimento. Ciência da Informação, 35(2), 27-38. Recuperado de http://www.scielo.br/pdf/\%0D/ci/v35n2/a04v35n2.pdf

Organisation for Economic Co-Operation and Development. (2007). OECD Principles and guidelines for access to research data from public funding. Paris. Recuperado de http://www.oecd.org/sti/inno/38500813.pdf

Oliveira, A. C. S.; Silva, E. M. (2016). Ciência aberta: dimensões para um novo fazer científico. Informação $\xi^{\prime}$ Informação, 21(2), 05-39. Recuperado de http://www.uel.br/revistas/uel/index.php/informacao/article/view/27666

Prodanov, C. C.; Freitas, E. C. de. (2013). Metodologia do Trabalho Científico: métodos e técnicas da pesquisa e do trabalho acadêmico (2a ed.). Novo Hamburgo: Feevale.

Sayão, L. S. F.; Sales, L. F. (2013). Dados de pesquisa: contribuição para o estabelecimento de um modelo de curadoria digital para o país. Tendências da Pesquisa Brasileira em Ciência da Informação, 6(1). Recuperado de http://www.brapci.inf.br/index.php/article/view/0000014157/0f3127bfd6475f45e6950 e1e0da20371

Sayão, L. F.; Sales, L. F. (2014). Dados abertos de pesquisa: ampliando o conceito de acesso livre. RECIIS: Revista Eletrônica de Comunicação, Informação \& Inovação em Saúde, Rio de Janeiro, 8(2),76-92. Recuperado de https://www.reciis.icict.fiocruz.br/index.php/reciis/article/view/611/1252

Sayão, L. F.; Sales, L. F. (2015). Guia de Gestão de Dados de Pesquisa para Bibliotecários e Pesquisadores. Rio de Janeiro: CNEN/IEN.

Targino, M. das G. (2000). Comunicação Científica: uma revisão de seus elementos básicos. Informação Es Sociedade, João Pessoa, 10(2), 67-85. Recuperado de http://www.periodicos.ufpb.br/ojs2/index.php/ies/article/view/326 